\title{
Safety, efficacy, and removability of a fully covered multi-hole metal stent in a swine model of hilar biliary stricture: a feasibility study
}

\section{(ㄷ)(우우}

Authors

Jin-Seok Park ${ }^{1,2}$, Seok Jeong ${ }^{1,2}$, Makoto Kobayashi ${ }^{3}$, Don Haeng Lee ${ }^{1,2,4}$

Institutions

1 Digestive Disease Center, Department of Internal Medicine, Inha University School of Medicine, Incheon, Republic of Korea.

2 National Center of Efficacy Evaluation for the Development of Health Products Targeting Digestive Disorders (NCEED), Incheon, Republic of Korea

3 Department of Gastroenterology, Yokkaichi Municipal Hospital, Yokkaichi, Japan

4 Utah-Inha DDS \& Advanced Therapeutics Research Center, Incheon, Republic of Korea

submitted 5.7.2018

accepted after revision 8.10 .2018

\author{
Bibliography \\ DOI https://doi.org/10.1055/a-0846-0775 | \\ Endoscopy International Open 2019; 07: E498-E503 \\ (c) Georg Thieme Verlag KG Stuttgart · New York \\ ISSN 2364-3722
}

\section{Corresponding author}

Seok Jeong, MD, Division of Gastroenterology, Department of Internal Medicine, Inha University Hospital, 27 Inhang-ro, Jung-gu, Incheon, 22332, Republic of Korea

Fax: +82-32-890-2549

inos@inha.ac.kr

\section{ABSTRACT}

Background and study aims Use of fully covered self-expandable metal stents (FCSEMS) for biliary drainage of malignant hilar biliary strictures is limited because of risk of cholangitis due to side branch obstruction. The aim of this study was to evaluate the safety and efficacy of unilateral placement of a multi-hole FCSEMS (FCSEMS-MH), which had a $2.5-\mathrm{mm}$ hole in the center of the membrane on each cavity, in a swine model of hilar biliary stricture.

Materials and methods Six female mini pigs were included in this prospective study. The hilar biliary stricture model was prepared using an endobiliary radiofrequency ablation. FCSEMS-MHs were endoscopically inserted into the peri-hilar bile duct of all the animals. Outcomes were assessed in terms of adverse events (AEs) including cholangitis, stent patency, stent migration, and endoscopic stent removability for 1 month after stenting.

Results In terms of hilar biliary stricture types, two animals were of Bismuth type I and four were of type II. Technical success rate for endoscopic metal stenting was $100 \%(6 / 6)$, and the functional success rate, which was evaluated 7 days after stent insertion, was also $100 \%$ (5/5). The immediate overall $\mathrm{AE}$ rate was $0 \%$. There was no procedure-related mortality and stent migration did not occur in any animal during the 4-week stent indwelling period. Stents were removed from all six animals at 4 weeks post-stenting.

Conclusion Unilateral placement of FCSEMS-MH might be safe and effect for swine hilar biliary stricture models. However, long-term follow-up and comparative studies are needed to verify the usefulness of this stent.

\section{Introduction}

Malignant hilar biliary obstruction is caused by a heterogeneous group of tumors, in which a limited number of patients are responsive to curative surgery [1]. The majority of patients with malignant hilar obstruction present with advanced disease, and endoscopic biliary stenting has become the principal palliative therapeutic option in such patients due to its minimal invasiveness, low cost, and relatively low morbidity and mortality [2]. Two types of stents, that is, plastic and uncovered self- expandable metal stent (SEMS), are routinely used in clinical practice. Metal stents are generally considered to remain patent for longer than plastic stents because they have larger lumens [3]. However, uncovered SEMSs are limited by recurrent biliary obstruction caused by tumor ingrowth and by difficulties associated with endoscopic reintervention [4,5]. Usually uncovered SEMSs are not removable a few days after deployment, because they become embedded in the tissue of the bile duct wall, which may totally block the mesh [6]. For occluded uncovered SEMS, inserting a second SEMS within the occlusion is fre- 
quently necessary because mechanical SEMS cleansing is not effective at restoring biliary patency [7]. To insert a second SEMS, a guidewire should be passed through the obstruction, which is sometimes extremely difficult to achieve. In our experience, removal of an occluded stent and reinsertion of a new stent at the stricture is easier in terms of procedural effectiveness and efficacy

On the other hand, a fully covered SEMS (FCSEMS) can be removed if required, and provides longer stent patency by preventing tissue ingrowth $[6,8]$. Therefore, FCSEMS might improve the efficacy of endoscopic drainage in cases of hilar biliary obstruction by increasing stent patency and procedural convenience. However, use of FCSEMS for treatment of hilar bile duct obstruction has not been recommended because of concerns regarding occlusion of smaller biliary branches joining the main duct at the hilum [1]. Accordingly, new FCSEMS designs are required for treatment of hilar biliary obstruction.

In this study, we evaluated the efficacy and safety of unilateral placement of a modified FCSEMS with holes in the center of each cavity into the peri-hilar bile duct, for bile drainage in a porcine model of hilar biliary obstruction.

\section{Materials and methods}

\section{Animals}

Six female miniature pigs (Sus scrofa; mean age 14 months, mean body weight $30 \mathrm{~kg}$ ) (Medikinetics Co., Pyeongtaek, Korea) were prepared for the in vivo experiments. Approval was obtained from our institutional animal care committee before study commencement (MK-IACUC 150803-001). All animals were quarantined and acclimated for 7 days before the experiments. All animal experiments were performed in NCEED large animal endoscopy laboratory. Animals were fasted but given water during the 24 hours before the endoscopic procedure, which was performed under general anesthesia. Pre-anesthesia sedation consisted of an intramuscular injection of tiletaminezolazepam $(5 \mathrm{mg} / \mathrm{kg})$, xylazine hydrochloride $(2 \mathrm{mg} / \mathrm{kg})$, and atropine sulfate $(0.04 \mathrm{mg} / \mathrm{kg})$. Animals were subsequently intubated, and general anesthesia was achieved with $1.5 \%$ isoflurane (Foran; JW Pharmaceutical Corp., South Korea). The procedures were conducted in the left decubitus position on a fluoroscopy table.

\section{Fully covered self-expanding metal stent with multi-holes}

The structure of the fully covered self-expanding metal stent with multi-holes (FCSEMS-MH) (M.I.Tech Co., Ltd, Pyeongtaek, Korea) is shown in > Fig. 1. The stent is constructed of nitinol wire and is completely covered with a silicone membrane on its inner surface. Uniquely, the stent has $2.5-\mathrm{mm}$ holes located in the center of membrane on each cavity, which prevents side branch obstruction and stent migration due to tissue ingrowth through the holes. The stent has a diameter of $10 \mathrm{~mm}$, a length of $3 \mathrm{~cm}$, and a round margin without flares at its ends. In addition, the stent has a $9 \mathrm{~cm}$-long lasso attached to its distal end for retrieval purposes.

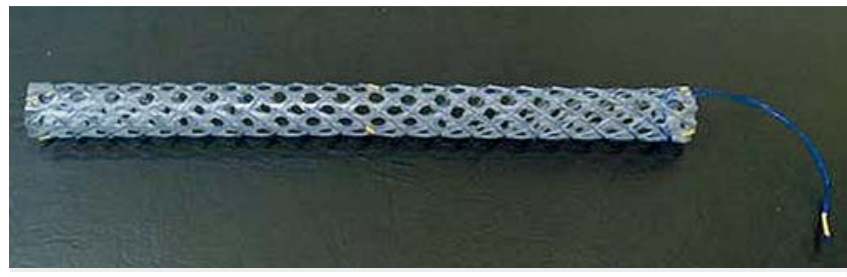

- Fig. 1 The FCSEMS-MH (M.I.Tech Co., Ltd, Pyeongtaek, Korea) has non-flared ends, a silicone covering membrane and a long lasso to facilitate stent retrieval. The stent has a unique design featuring 2.5-mm holes located in the center of the membrane of each cavity, which prevent bile duct side branch obstruction and stent migration.

\section{Hilar bile duct stricture model}

All endoscopic procedures were performed by an expert biliary endoscopist (S. J.) using a standard side-viewing duodenoscope (TJF-240; Olympus Optical Co. Ltd., Tokyo, Japan). After duodenal intubation of the scope, diagnostic cholangiography was achieved using a diagnostic cannula (endoscopic retrograde cholangiopancreatography [ERCP] catheter, bottle-shaped metal tip; MTW-Endoskopie, Wesel, Germany), which was inserted into the bile duct using the wire-guided cannulation technique and a 0.035-inch hydrophilic-tipped guidewire (Boston Scientific Corporation, Natick, Massachusetts, United States). An endobiliary radiofrequency ablation (RFA) catheter (18 mm electrode length, 7F diameter, 175-cm working length; STARmed Co., Ltd, Goyang, Korea) was then advanced into the proximal common bile duct (CBD) or common hepatic duct (CHD) over the guidewire under fluoroscopic guidance. RFA applications (VIVA combo RF generator, VCS10, STARmed Co., Ltd) were performed at an energy setting of $7 \mathrm{~W}, 80^{\circ} \mathrm{C}$ for 120 seconds. RFA at the proximal CBD or CHD was performed with the intention to create a Bismuth type I or type II hilar biliary stricture, respectively, assuming stricture length was about 10- mm. During the 4 weeks following RFA application, animals were fed their routine diet. Clinical signs and parameters, such as weight loss and daily food intake, were monitored daily. Liver function tests were performed before and 4 weeks after the procedure. Cholangiography was repeated 4 weeks after RFA application to identify hilar bile duct strictures. Bile duct stricture was defined as a luminal diameter decrease of $50 \%$ or more than the baseline at the RFA application site and presence of intrahepatic bile duct dilatation.

\section{Endoscopic placement of the FCSEMS-MH}

ERCP was performed using a duodenoscope (TJF-240; Olympus Optical Co. Ltd., Tokyo, Japan) under general anesthesia. After obtaining a cholangiogram, a FCSEMS-MH was inserted over a guide wire using an 8-Fr delivery system under fluoroscopic guidance and released at the site of stricture. Endoscopic biliary sphincterotomy was not required for stent insertion because unlike humans, swine do not have sphincter of Oddi in major duodenal papilla, and the papillary orifice is patulous and wide. Technical success was defined as appropriate stent posi- 

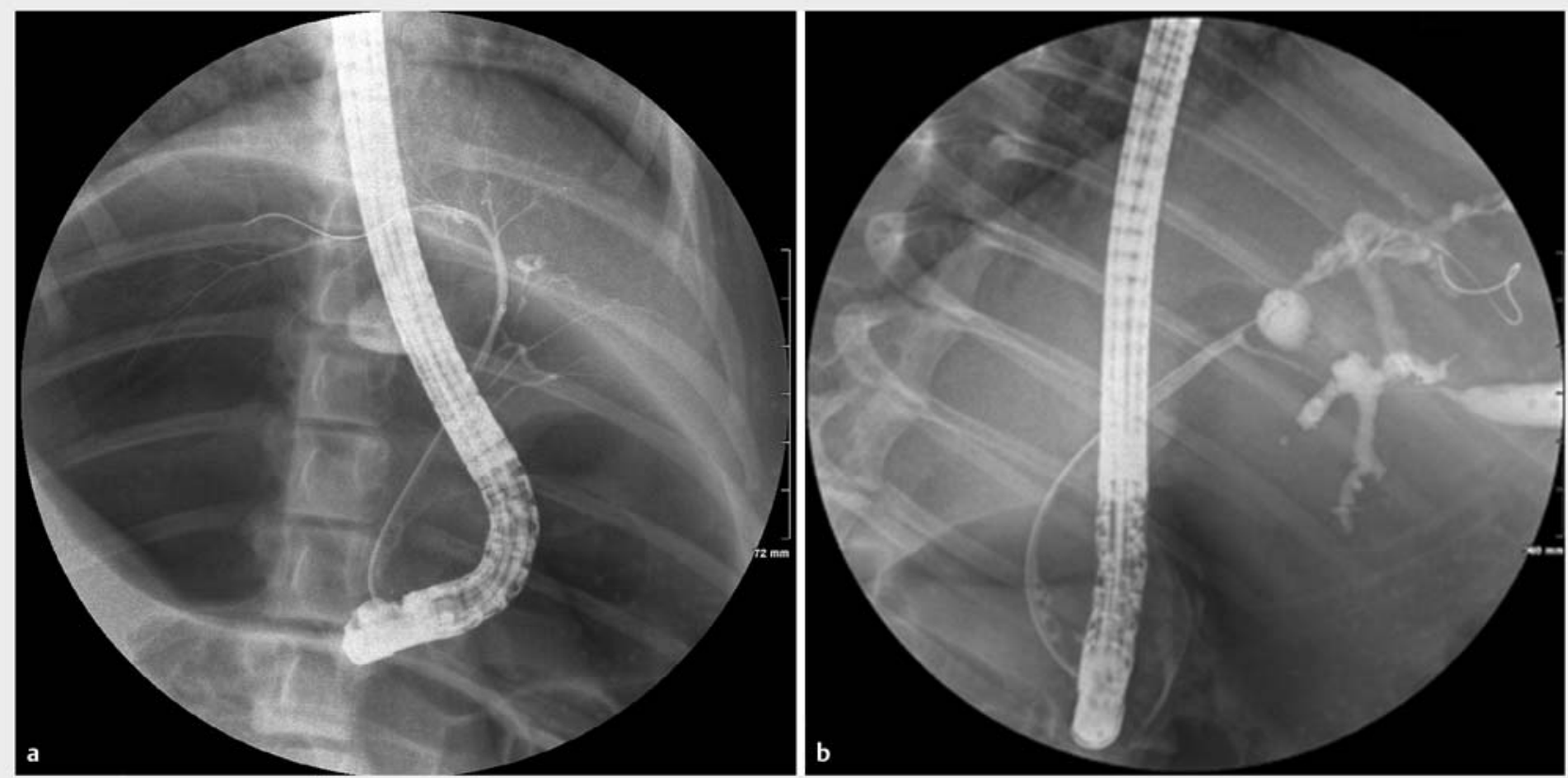

- Fig. 2 Endoscopic retrograde cholangiogram. a Normal cholangiogram before endobiliary radiofrequency ablation (RFA). b Cholangiogram obtained at 4 weeks after endobiliary RFA showing segmental stricture of the common hepatic duct with intrahepatic duct dilatation.

tioning along the stricture with free flow of contrast through the deployed stent.

\section{Follow-up evaluation and stent removal}

For 4 weeks after stent insertion, clinical evaluation was performed daily to confirm improvements in obstructive jaundice and detect adverse events (AEs). Clinical signs and parameters including weight loss, daily food intake, and demeanor score were monitored. Biochemical liver function tests were performed at baseline and weekly after stent insertion. Clinical success was defined as a reduction in serum bilirubin level of $>50 \%$ during the first week after stent placement. AEs including acute cholecystitis, stent migration, and stent occlusion were evaluated. Stents were removed at 4 weeks in all animals to evaluate their removability. A rat tooth forceps was used to grasp and pull back the lasso at the distal end of stent to remove the stent.

\section{Results}

\section{Development of the hilar biliary stricture model}

Endoscopic RFA of the peri-hilar bile duct was successfully performed on all six animals. No technical difficulties or AEs were encountered during creation of the biliary stricture model, and no clinical evidence of a procedure-related AE, such as, cholangitis, cholecystitis, perforation, and bleeding, was obtained during the 4 weeks the stents were in place. On follow-up ERCP, which was performed at 4 weeks after RFA, segmental stricture of peri-hilar bile duct and both intrahepatic duct dilatation was observed in all animals ( $\boldsymbol{F}$ Fig. 2). Based on laboratory test results, definite obstructive jaundice was observed in

\begin{tabular}{l|c|}
$\begin{array}{l}\text { Table } 1 \text { Baseline characteristics of hilar biliary stricture model, pre- } \\
\text { stenting. }\end{array}$ & 6 \\
\hline \begin{tabular}{l} 
The location of stricture, $\mathrm{n}$ \\
\hline $\begin{array}{l}\text { - Common hepatic duct } \\
\text { (Bismuth-Corlette type I) }\end{array}$
\end{tabular} & 2 \\
\hline $\begin{array}{l}\text { - The confluence of right and left hepatic } \\
\text { duct (Bismuth-Corlette type II) }\end{array}$ & 4 \\
\hline \begin{tabular}{l} 
The occurrence of obstructive jaundice, $\mathrm{n}(\%)$ \\
\hline
\end{tabular} & $5(83.3)$ \\
\hline \begin{tabular}{l} 
Liver enzyme, median (range) \\
\hline - Total bilirubin, mg/dL
\end{tabular} & $4.7(0.2-8.26)$ \\
\hline - Aspartate aminotransferase, IU/L & $76.6(39.1-341.9)$ \\
\hline - Alanine aminotransferase, IU/L & $22.4(11.5-26.3)$ \\
\hline
\end{tabular}

five animals, but cholestasis did not occur in one animal with a CHD stricture ( $\triangleright$ Table 1$)$. Median liver profile values were as follows: total bilirubin, $4.7 \mathrm{mg} / \mathrm{dL}$ (range, $0.2-8.26$ ); aspartate aminotransferase, 76.6 IU/L (39.1-341.9); alanine aminotransferase, $22.4 \mathrm{IU} / \mathrm{L}(11.5-26.3)$.

\section{Outcomes of stenting and stent removal}

Intraductal placement of the FCSEMS-MH was technically successful in all animals. All stents were positioned across the stricture and expanded immediately to maximal diameter in all cases ( $\triangleright$ Fig. 3). Clinical success with stenting was achieved in $100 \%(5 / 5)$ of animals with obstructive jaundice caused by biliary RFA ( $\triangleright$ Table 2). Early AEs related to stenting, such as, cholangitis, perforation, and bleeding, were not encountered in 

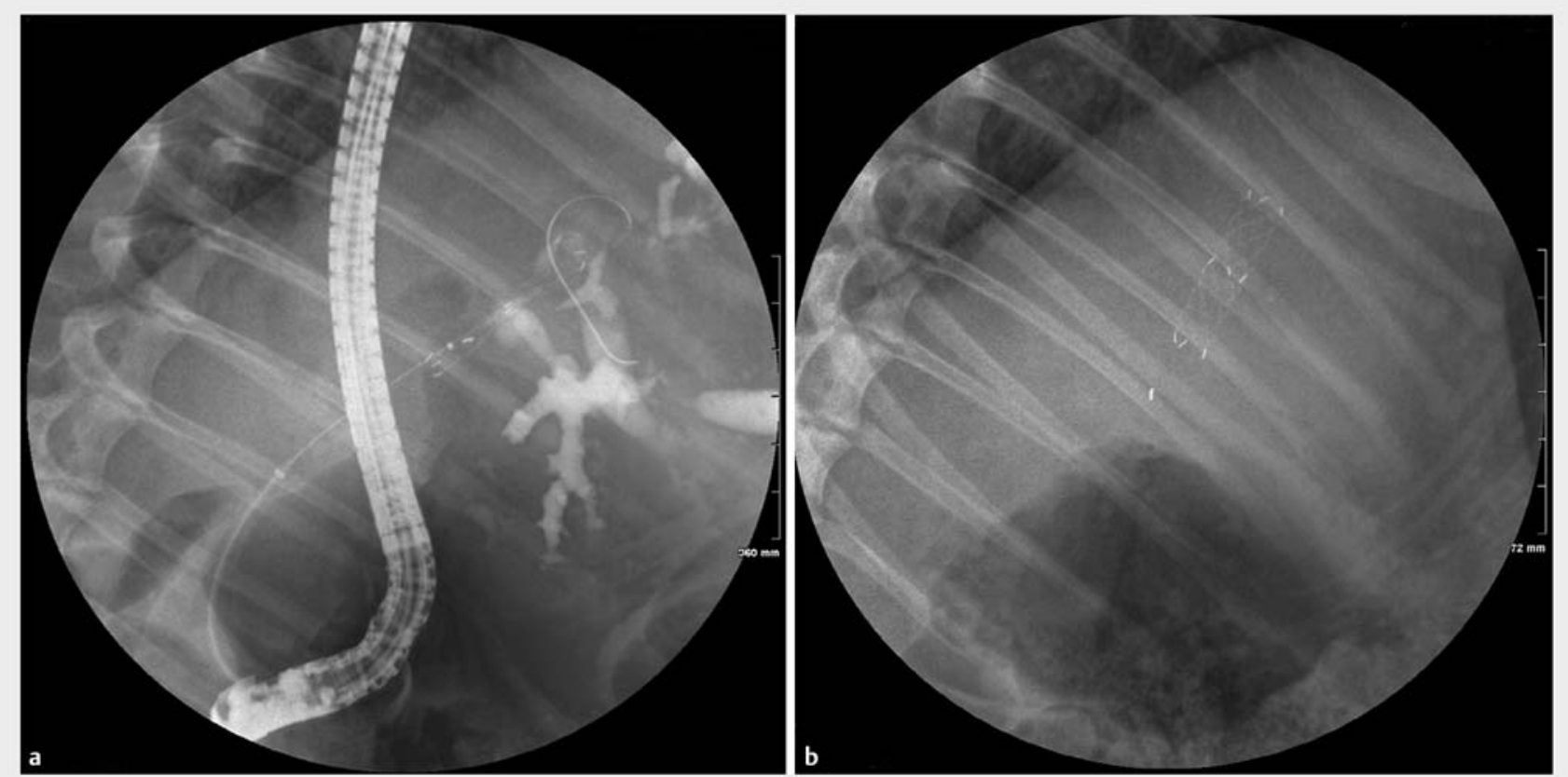

- Fig. 3 Unilateral placement of the FCSEMS-MH in the hilar biliary stricture model. a Fluoroscopic finding right after placement of the FCSEMS-MH. b Fully expanded the FCSEMS-MH.

- Table 2 Overall outcomes of FCSEMS-MH placement.

Outcome measures

\begin{tabular}{|l|c|}
\hline Technical success, (\%) & $6 / 6(100)$ \\
\hline Functional success, (\%) & $5 / 5(100)$ \\
\hline \multicolumn{2}{|l|}{ Liver enzyme, 4 weeks after stenting, median (range) } \\
\hline
\end{tabular}

\begin{tabular}{|l|l|}
\hline - Total bilirubin, $\mathrm{mg} / \mathrm{dL}$ & $1.2(0.2-1.4)$ \\
\hline - Aspartate aminotransferase, IU/L & $80.2(40.4-345.3)$ \\
\hline - Alanine aminotransferase, IU/L & $23.0(11.5-31.2)$ \\
\hline Adverse events & \\
\hline - Cholangitis (\%) & $0 / 6(0)$ \\
\hline - Stent migration (\%) & $0 / 6(0)$ \\
\hline - Stent dysfunction (\%) & $0 / 6(0)$ \\
\hline Success of stent removal (\%) & $6 / 6(100)$ \\
\hline
\end{tabular}

FCSEMS-MH, fully covered self-expanding metal stent with multi-holes

any of the six animals. During the 4-week stent indwelling period, no stent occlusion or stent migration occurred in any animal ( Fig.4). In addition, cholangitis, which can be caused by side branch obstruction, did not occur. Endoscopic FCSEMS-MH removal was successful and performed without difficulty in all animals, and after removal, no AEs were encountered. In all cases, stent removal was achieved without resistance by pulling the lasso into the working channel ( $\triangleright$ Fig.4).

\section{Discussion}

This pilot study shows that FCSEMS-MH placement offers a potentially effective palliative treatment for malignant hilar biliary stricture. In fact, technical and functional success rates were both $100 \%$. In addition, the study demonstrates that cholangitis induced by FCSEMS placement in the hilar bile duct can be prevented by making multiple holes in the covering membrane of stent cavities to prevent side branch occlusion. Results of this study suggest that this FCSEMS modification is reasonable for treatment of hilar biliary stricture.

Endoscopic biliary stenting is an effective management modality for malignant hilar biliary strictures and remains firstline palliative treatment for relieving jaundice, preventing cholangitis, and improving quality of life $[9,10]$. Placement of an FCSEMS for malignant biliary strictures has some merits as compared with uncovered SEMS, that is, longer stent patency and straightforward stent removal according to clinical need $[3,11]$. However, FCSEMSs are not indicated for hilar biliary obstruction because they may occlude the contralateral biliary system or biliary side branches. In a previous study, placement of a plastic stent in the contralateral intrahepatic duct to facilitate bile flow before fully covered SEMS placement was investigated by Poley et al. [12]. In this study, a FCSEMS in conjunction with a contralateral $10 \mathrm{Fr}$ plastic stent was placed in the hilar biliary stricture. The authors concluded that placement of the plastic stent effectively prevented cholangitis induced FCSEMS insertion [12]. However, theoretically, plastic stent insertion is insufficient to address risk of cholangitis due to side branch occlusion. In addition, a plastic stent should be exchanged on a regular basis, which would be a burden for a patient with malig- 

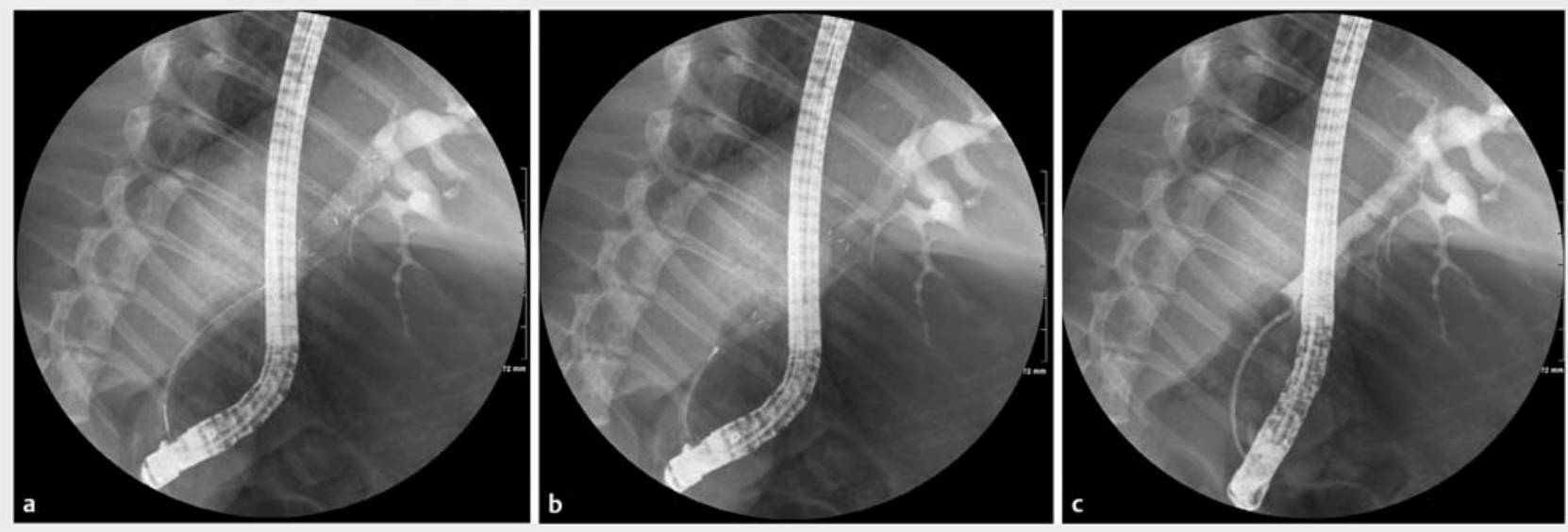

- Fig. 4 Fluoroscopic finding 4 weeks after FCSEMS-MH placement. a The FCSEMS-MH remained at its insertion location without evidence of migration and intrahepatic duct dilatation was improved. b Fluoroscopic view showing endoscopic removal of the FCSEMS-MH 4 weeks after placement. c Cholangiogram performed after stent removal showing improvement of hilar biliary stricture and intrahepatic duct dilatation.

nant hilar biliary stricture. To address this issue, we made multiple holes in the FCSEMS-MH membrane in the center of each mesh to maintain bile flow between the contralateral bile duct and side branches. Our results showed the FCSEMS-MH achieved our functional targets in the five pigs with obstructive jaundice without cholangitis in any animal. Therefore, this new stent design is expected to be effective in treating hilar malignant stricture involving the hepatic duct confluence with unilateral biliary stenting by reducing risk of that obstructive cholangitis will obstruct the opposite part of hepatic duct. However, it should not be expected that unilateral stenting with this stent would prevent cholangitis in the case of a stricture that extends into the bilateral proximal hepatic ducts up to the segmental bile duct (Bismuth-Corlette classification type IV). For such strictures, bilateral stent insertion, such as in side-byside insertion, would be necessary to achieve successful biliary drainage. Furthermore, this stent application may not be appropriate in cases in which the hilar stricture extends up to the segmental bile duct. Since the diameter of segmental bile duct is relatively smaller than that for the hepatic duct, the stent cannot expand sufficiently and the segmental bile duct can be easily obstructed by covering the membrane or insufficiently expanded stent.

Stent migration is a primary issue when a FCSEMS is used to treat biliary stricture $[3,7]$, and has been reported to occur more frequently in patients that receive a FCSEMS than other types of SEMS $[8,13]$. Most FCSEMSs usually have flared ends to prevent stent migration. However, we conjectured that the flared end might induce injury to the normal bile duct wall and cause embedment into the bile duct and/or tissue hyperplasia leading to removal difficulties and limited stent patency [14]. The newly modified FCSEMS used in the current study has nonflared ends to reduce injury to the bile duct wall. Stent migration was not encountered during the current study, and we believe that was due to the low tension of covered membrane associated with the small holes. This low tension of membrane induces tissue to bump into each cavity of the stent in a concave fashion, but it does not allow the metallic wire to become embedded in the underlying tissue. This bump action also acts as natural anchoring. In addition, malignant tissue budding grows from the bile duct wall into the stent lumen through the hole with the passage of time and the anti-migration effect of this stent would be expected to increase. However, because histologic evaluation of bile ducts was not performed after the FCSESM-MH placement, this concept of natural anchoring could not be verified by laparotomy. Nevertheless, given the absence of cholangitis or stent migration in the experiments, we anticipate that holes introduced in the membrane of covered stents would facilitate palliative management of patients with malignant hilar biliary stricture by providing prolonged stent patency.

Biliary stenting with an FCSEMS enables stent removal according to clinical needs [15]. Uncovered SEMS usually cannot be removed after placement, but occlusion of an uncovered SEMS usually can be managed endoscopically by placing a second biliary stent in a stent-in-stent fashion [16]. However, revision of the occluded stent is more technically challenging than first stent insertion because the guidewire is often inserted improperly outside of the stent lumen through the stent cavities, and cannot be passed through the stent lumen from the distal to proximal ends. In addition, revised endoscopic stenting with a second stent through the first stent lumen may result in a shorter period of patency for the stent than for the first one placed because of the reduced effective diameter. Therefore, replacement of an occluded sent with a new SEMS offers a better treatment strategy in terms of its efficacy and technical merits.

The current study has several limitations that warrant consideration. First, it is limited by its non-comparative design and small sample size. Because the aim of this study was to evaluate feasibility and safety of the FCSEMS-MH in a swine model of hilar biliary stricture, the study design was suboptimal 
with respect to proving the efficacy of FCSEMS-MH in patients with hilar malignant biliary stricture. Further prospective comparative studies are required to confirm the effectiveness of FCSEMS-MHs in patients with hilar malignant biliary stricture. Second, this study was conducted using a porcine model of benign biliary stricture, and although animal-based efficacy and safety evaluations of developed stents are essential before clinical trials, there is no suitable large animal model for malignant biliary stricture, and thus, we used a benign biliary stricture model. As a result, experimental evidence obtained in this in vivo study is probably insufficient to support the clinical utility of FCSEMS in patients with malignant biliary stricture. Third, the stent indwelling period (1 month) was insufficient to show long-term outcomes. Long-term outcomes, such as risk of stent dysfunction and stent embedding due to tumor ingrowth through the holes in the stent-covering membrane, could not be evaluated in our experimental model. Because the aim of the current study was to evaluate the feasibility of FCSEMS-MH in hilar obstruction, we focused on development of cholangitisrelated stent insertion in the early phase after stent insertion. Therefore, further study is warranted with a longer indwelling period to determine whether stent function can be maintained for long time.

\section{Conclusion}

In conclusion, unilateral placement of a FCSEMS-MH in hilar biliary strictures was found to be feasible and acceptable in terms of managing cholestasis and preventing stent migration and cholangitis. Furthermore, FCSEMS-MH stent removal was straightforward after a 1-month stent-indwelling period. Therefore, this newly designed FCSEMS may facilitate preoperative and palliative management of patients with hilar malignant biliary stricture. Long-term follow-up and comparative human studies are required to confirm the efficacy of the FCSEMS-MH for treatment of malignant hilar biliary stricture.

\section{Acknowledgements}

This work was supported by an Inha University Hospital research grant.

\section{Competing interests}

None

\section{References}

[1] Larghi A, Tringali A, Lecca PG et al. Management of hilar biliary strictures. Am J Gastroenterol 2008; 103: 458-473

[2] Ducreux M, Liguory C, Lefebvre J et al. Management of malignant hilar biliary obstruction by endoscopy results and prognostic factors. Dig Dis Sci 1992; 37: 778-783

[3] Dumonceau J, Tringali A, Blero D et al. Biliary stenting: indications, choice of stents and results: European Society of Gastrointestinal Endoscopy (ESGE) clinical guideline. Endoscopy 2012; 44: 277-298

[4] Shim C, Lee Y, Cho Y et al. Preliminary results of a new covered biliary metal stent for malignant biliary obstruction. Endoscopy 1998; 30 : $345-350$

[5] Isayama H, Mukai T, Itoi T et al. Comparison of partially covered nitinol stents with partially covered stainless stents as a historical control in a multicenter study of distal malignant biliary obstruction: the WATCH study. Gastrointest Endosc 2012; 76: 84-92

[6] Isayama H, Komatsu Y, Tsujino T et al. A prospective randomised study of "covered" versus "uncovered" diamond stents for the management of distal malignant biliary obstruction. Gut 2004; 53: 729 734

[7] Kahaleh M, Talreja JP, Loren DE et al. Evaluation of a fully covered selfexpanding metal stent with flared ends in malignant biliary obstruction: a multicenter study. J Clin Gastroenterol 2013; 47: e96-100

[8] Kasher JA, Corasanti JG, Tarnasky PR et al. A multicenter analysis of safety and outcome of removal of a fully covered self-expandable metal stent during ERCP. Gastrointest Endosc 2011; 73: 1292 - 1297

[9] Larghi A, Tringali A, Lecca PG et al. Management of hilar biliary strictures. Am J Gastroenterol 2008; 103: 458-473

[10] Siddiqui AA, Mehendiratta V, Loren D et al. Self-expanding metal stents (SEMS) for preoperative biliary decompression in patients with resectable and borderline-resectable pancreatic cancer: outcomes in 241 patients. Dig Dis Sci 2013; 58: $1744-1750$

[11] Tarantino I, Mangiavillano B, Di Mitri R et al. Fully covered self-expandable metallic stents in benign biliary strictures: a multicenter study on efficacy and safety. Endoscopy 2012; 44: 923 - 927

[12] Poley J, van Tilburg AJ, Kuipers EJ et al. Breaking the barrier: using extractable fully covered metal stents to treat benign biliary hilar strictures. Gastrointest Endosc 2011; 74: 916 - 920

[13] Johanson JF, Schmalz MJ, Geenen JE. Incidence and risk factors for biliary and pancreatic stent migration. Gastrointest Endosc 1992; 38: $341-346$

[14] Moon S, Kim M, Park DH et al. Modified fully covered self-expandable metal stents with antimigration features for benign pancreatic-duct strictures in advanced chronic pancreatitis, with a focus on the safety profile and reducing migration. Gastrointest Endosc 2010; 72: 86-91

[15] García-Cano J. Use of fully covered self-expanding metal stents in benign biliary diseases. World J Gastrointest Endosc 2012; 4: 142 - 147

[16] Ridtitid W, Rerknimitr R. Management of an occluded biliary metallic stent. World J Gastrointest Endosc 2012; 4: 157-161 